\title{
Evaluation of Yield and Yield Related Traits of Chickpea (Cicer arietinum L.) Genotypes under Water Stress Condition
}

\author{
R. T. Shende ${ }^{1 *}$, R. S. Sengar ${ }^{1}$, Mukesh Kumar ${ }^{1}$, Shalani Gupta ${ }^{2}$, \\ Gopal Singh ${ }^{3}$ and S. K. Singh ${ }^{4}$
}

${ }^{1}$ Department of Agriculture Biotechnology, ${ }^{2}$ Department of Molecular Biology \& Genetic Engineering, ${ }^{3}$ Department of Plant Pathology, ${ }^{4}$ Department of Genetics and Plant Breeding, College of Agriculture, Sardar Vallabhbhai Patel University of Agriculture and Technology, Modipuram, Meerut, India

*Corresponding author

\section{A B S T R A C T}

\begin{tabular}{|l|}
\hline Ke y w o r d s \\
Chickpea, Water \\
stress, \\
Morphological, \\
Yield trait
\end{tabular}

Keywords

\section{Introduction}

Chickpea (Cicer arietinum $\mathrm{L}$; $2 \mathrm{n}=2 \_=16$ ), a member of the Fabaceae, is the most essential legume crop after dry beans (Phaseolus vulgaris) and dry pea (Pisum sativum) with a genome size of $\sim 738 \mathrm{Mb}$ and 28269 genes (Varshney et al., 2013, Parthasarathy Rao et al., 2010, Pandey et al., 2018). Chickpea (Cicer arietinum L.) belong to Leguminosae family and Cicer genus consist of 43 mostly perennials species and 9 species those are annuals (Pundir et al., 1985). In India, Chickpea cultivated almost in all parts of the country mainly as a rainfed crop (68\% area) (DAC, Agricultural Statistics at a Glance 2019). During 2018-19, chickpea production has been estimated to be about 8.93 million tonnes (mt), total area 9.35 million hectares with an average yield is $955 \mathrm{Kg}$. /hectare, which is $46 \%$ of the total pulses production (23.95 mt) in India ((DAC, Agricultural Statistics at a Glance 2019). Due to nitrogenfixing root nodulation capacity Chickpea is 
valuable crop for improvement of soil fertility especially in rainfed or dry regions (Katerji et al., 2001, Kumar, S. 2016). Chickpea seed contains $17-31 \%$ protein it and biological activity of protein ranged from 52 to $78 \%$ has a prime significance for human food and animal feed. Essential amino acids like leucine, isoleucine, lysine, valine and phenylalanine are present in significant amount in the readily digestible seed protein (Williams and Singh 1980, Karim and Fattah 2006).

In globally chickpea can be divided into two grouped, that is 'Kabuli' and 'Desi'. Kabuli' type has large seeds with a thin and light coloured seed coat, grown mainly in the Mediterranean regions and used as whole seed in foods. 'Desi' type of chickpea is grown mainly in the Indo-Pak subcontinent and Ethiopia (Pundir et al., 1985). About 52 countries grow chickpea including several in North and East Africa, West and South Asia, Australia and South Europe (Ganjeali et al.2011). It is grown either as a dry weather crop in semi-arid zones or as a rainfed crop in cool climates (Tahir and Karim 2010). In practice, about $90 \%$ chickpea crop is grown in rainfed conditions without reliance on irrigation (Kumar and Abbo, 2001) and thus both vegetative and reproductive phases are adversely affected by water deficiency.

Plants are subjected to various stress conditions in their life cycle. Drought is the second major constraint on chickpea productivity after diseases (Singh et al.1994).

Drought limits the agricultural production by preventing the crop plants from expressing their full genetic potential. Many researchers believed that tolerance to drought stress must be done via genetic improvement of seed yield in crops (Passioura, 1996). Different workers used different methods to evaluate genetic differences in drought tolerance
(Bidinger et al., 1982). Breeding for drought tolerance is generally considered slow due to the quantitative and temporal variability of available moisture across years, the low genotypic variance in yield under these conditions, inherent methodological difficulties in evaluating component traits, together with the highly complex genetic basis of this character. Selection for drought resistance and production of tolerant cultivars with high yield potential is the main objective of breeding programmes.

Several morphological traits have been listed to play a significant role in crop adaptation to drought stress (Saxena and Johansen 1990; Subbarao et al., 1995). In addition, information on the seed yield, number of pods per plant, number of seeds per pod, 100-seed weight and root traits and the phenotypic correlation among these traits will be useful for planning suitable breeding strategies to improve drought tolerance. The effective selection for traits under improvement depends on sufficient additive genetic variation of the traits. Phenotypic relationships among these traits are also important when simultaneous selection of multiple traits is to be carried out for high yield under drought stress conditions (Painawadee et al., 2009).

\section{Materials and Methods}

\section{Experimental plan and water stress treatment}

The present experiment was conducted during Nov-2018 to April-2019 and Nov-2019 to April-2020. Ten chickpea genotypes were collected for present investigation from IIPR, Kanpur, NBPGR, New Delhi and MPKV, Rahuri. India. The description of chickpea genotype is given in Table 1. Each genotype was planted in plastic pots $\left(39 \times 33 \mathrm{~cm}^{2}\right.$ pot containing $25.0 \mathrm{~kg}$ of soil and $5.0 \mathrm{~kg}$ FYM) in 
completely randomized design, with three biological replications for each genotype. Ten seeds of each genotypes were planted in each pot. The experimental soil was sandy loam with initial $\mathrm{pH} 7.2$ and ECe of $1.39 \mathrm{dSm}^{-1}$. After 18 days of planting, thinning was carried out and only three plants were left in each pot for further experiment. Similar management inputs like fertilizer and insecticides for proper growth and disease control were given during the experimental period. The water stress was applied by irrigation suppression during Vegetative stage (40 DAS). The plants were constantly irrigated till $40^{\text {th }}$ day of planting. The stress was imposed by withholding irrigation for a period of 7-8 days at above stage. Visual appearance of wilting during morning hours was taken the criterion for the stress and take morphological observations.

\section{Weather condition}

The meteorological observations were recorded by an automatic weather station of Indian Institute of Farming System and Research (IIFSR), Modipuram, Meerut, India. The recorded observation are presented in following figure and table (Table 2 and Fig. 1). The observation recorded were min and max temp, percent relative humidity in morning and evening, average rainfall and bright sunshine for Nov-2018 to May-2019 and Nov-2019 to May-2020

\section{Observation of morphological trait}

Morphological trait was recorded after stress treatment in drought stressed and control plants. The traits Viz., days to 50\% flowering, plant height, number of branches, number of pods per plant, 100 seed weight, yield per plant were recorded for three randomly selected plants for each replication for morphological trait analysis.

\section{Statistical analysis}

All the above data were recorded in triplicate \pm SD and analyzed statistically using completely randomized design (Panse and Sukhatme, 1970). Graph pad prism version 5.0 were used to performed graph of results.

\section{Results and Discussion}

\section{Meteorological observations}

The meteorological variables during the experimental period from week $1^{\text {st }}$ to week $17^{\text {th }}$ (Nov-2018 to April-2019) are presented in Fig. 2 and Table 3. During the whole experimental period, the weekly min temperature ranged from 5.9 to 23.3 with a general mean of $11.4^{\circ} \mathrm{C}$ whereas, max temperature ranged from 15.4 to 40.7 with a grand mean of $27.7^{\circ} \mathrm{C}$. Total rainfall received was $39.3 \mathrm{~mm}$, relative humidity varies from 57.9 to 96.8 having a general mean of $85.7 \%$ in morning wherein evening time it varied from 17.6 to 69.0 with an average of $38.9 \%$ in evening and $171.2 \mathrm{~h}$ of bright sunshine (BSS) during entire experimental period.

\section{Effect of water stress on growth and yield attributes}

The data pertaining to various yield attributes of chickpea crop viz. branches per plant, pods per plant, test weight and yield per plant are presented in Table 3.

\section{Days to 50 per cent flowering}

The data reveals that amongst the control and drought environment, significantly highest days to 50 per cent flowering plants ${ }^{-1}$ were recorded in control during both the years. With drought stress under terminal moisture stress there was a significant and concomitant reduction in the Days to 50 per cent flowering plants $^{-1}$ during both the years. The detailed 
results evaluated under control condition as well as stress condition for both the years are represented in Table 3 and 4 respectively.

In Rabi season 2018-2019 among the genotypes, under non-stress condition maximum number of Days to 50 per cent flowering plants ${ }^{-1}$ was recorded in GNG-469 (76 \pm 2.08) followed by DCP-92-3 (71 \pm 1.15), Pusa-362 (70 \pm 0.88$)$ and JG $11(63 \pm$ 1.15) while minimum number of Days to 50 per cent flowering plants $^{-1}$ was recorded in shows in Annigeri $1(47 \pm 1.15$ whereas, under stress condition maximum number of Days to 50 per cent flowering plants $^{-1}$ was recorded in GNG 469 (71.33 \pm 0.66), followed by DCP-92-3 (66 \pm 1.52$)$, Pusa-362 (3.6), and JG 11 (58.33 \pm 0.88$)$ while minimum number of Days to 50 per cent flowering plants ${ }^{-1}$ was recorded in recorded in Vishal (43.33 \pm 1.76$)$ (Table 3). Maximum reduction precent was recorded in Vishal $(12.75 \%)$ and minimum was recorded in ICC $4958(4.90 \%)$ followed by ICC $867(5.10 \%)$ (Table 5). Therefore, the range from 47 to 76 and 43.33 to 71.33 Days to 50 per cent flowering plants $^{-1}$ under non-stress and stress condition, respectively (Figure 2).

But, Rabi season 2019-2020 data w.r.t. Days to 50 per cent flowering plants $^{-1}$ were having variation over the first year this variation w.r.t. number of Days to 50 per cent flowering plants $^{-1}$ in both the year, may be due to environmental effects. The results revealed that Days to 50 per cent flowering plants $^{-1}$ decreases significantly with water stress in all the ten varieties. Maximum percent reduction was recorded in Vishal $(21.85 \%)$ while the minimum percent reduction was observed in ICC 4958 (4.48\%) (Table 6). Result further showed under non stress condition variety GNG-469 (74.66 \pm 0.88$)$ recorded the maximum number of Days to 50 per cent flowering plants $^{-1}$ closely followed by Pusa-362 (71.33 \pm 0.88$)$,
DCP-92-3 (68.33 \pm 0.88$)$ and JG $11(61.33 \pm$ 0.88) (Table 4) whereas, the minimum number of Days to 50 per cent flowering plants $^{-1}$ was recorded in ICC 4958 (44.66 \pm 1.45) While under water stress condition variety Pusa-362 $(66 \pm 1.52)$ showed the maximum number of Days to 50 per cent flowering plants ${ }^{-1}$ closely followed by GNG469 (65 \pm 1.73$)$, DCP-92-3 (64.33 \pm 2.33$)$ and JG $11(57.33 \pm 2.33)$ whereas the minimum number of Days to 50 per cent flowering plants $^{-1}$ was recorded in Vishal $(39.33 \pm 1.76)$ (Figure 3). Therefore, the range from 44.66 to 74.66 and 39.33 to 66 under non-stress and stress condition, respectively. ANOVA results have indicated that the number of Days to 50 per cent flowering plants $^{-1}$ is found to be significant (Figure 4).

In the present study, significant difference were observed among the genotypes with respect to days to 50 percent flowering. Among the genotypes GNG 469 (76 days) and Annigeri 1 (47 days); ICC 4958 (44.66 days) had taken less days for days to 50 percent flowering in both experimental years. Similarly, Mathur and Mathur (1996) and Yadav et al., (2001) also reported that days to 50 percent flowering negatively correlated with seed yield under moisture stress. Ahmed et al., (2011) also reported range 41.03-72.33 in control and 41.0 to 72.33 drought stress to the days to 50 per cent flowering.

\section{Plant height (CM)}

Plant height (PH) w.r.t. different genotype was influenced by the environment/climatic condition. The detailed results evaluated under control condition as well as stress condition for both the years are represented in Table 3 and 4 at harvest time, respectively.

It is revalued as plant as plant growth progressed, plant height increased and it was positively correlated at different plant growth 
stages. There was genetic variation with respect to plant height among the genotypes.

Rabi season 2018-2019 plant height differs significantly with respect to treatment. The results revealed that plant height decreases significantly with water stress in all the ten varieties. Maximum reduction was recorded in Vishal (26.54\%) while the minimum reduction was observed in ICC $4958(6.38 \%)$ followed by ICC 867 (8.27\%) (Table 5). Result further showed under non stress condition variety Pusa $362(86.43 \pm 1.30 \mathrm{~cm})$ recorded the maximum plant height closely followed by GNG-469 $(84.06 \pm 1.28 \mathrm{~cm}), \mathrm{JG}$ 11 (77.06 \pm 1.12) and Annigeri 1 (74.78 \pm $1.23 \mathrm{~cm})$ whereas, the minimum plant height was recorded in Digvijay $(52.11 \pm 0.73 \mathrm{~cm})$. While under water stress condition variety GNG-469 $(75.46 \pm 0.52 \mathrm{~cm})$ showed the maximum plant height closely followed by Pusa $362(71.91 \pm 1.48 \mathrm{~cm})$, Annigeri 1 $(69.41 \pm 0.75 \mathrm{~cm})$ and JG $11(63.047 \pm 1.55$ $\mathrm{cm})$ whereas the minimum plant height was recorded in Vishal $(42.79 \pm 1.20 \mathrm{~cm})$. Therefore, the range from $52.11 \pm 0.73 \mathrm{~cm}$ to $86.43 \pm 1.30 \mathrm{~cm}$ and $42.79 \pm 1.20 \mathrm{~cm}$ and $75.46 \pm 0.52$ under non-stress and stress condition respectively (Figure 4).

But, the Rabi season 2019-2020 data w.r.t. plant height were having variation over the first year this variation w.r.t. plant height in both the year, may be due to environmental effects. The results revealed that plant height decreases significantly with water stress in all the ten varieties. Maximum reduction was recorded in Vishal $(11.49 \%)$ and minimum reduction was recorded in ICC $4958(2.74 \%)$ followed by ICC $867(5.02 \%)$ (Table 6). Result further showed under non stress condition variety GNG $469(86.57 \pm 1.08 \mathrm{~cm})$ recorded the maximum plant height closely followed by Pusa $362(80.94 \pm 1.07 \mathrm{~cm})$ and ICC $4958(75.73 \pm 0.13 \mathrm{~cm})$ whereas the minimum plant height was recorded in ICC
$867(42.14 \pm 1.08 \mathrm{~cm})$. While under water stress condition variety GNG (74.76 \pm 1.20 $\mathrm{cm})$ showed the maximum plant height closely followed by ICC 4958 (72.98 \pm $1.44 \mathrm{~cm})$, and Pusa $362(70.43 \pm 1.03 \mathrm{~cm})$, and whereas the minimum plant height was recorded in ICC $867(37.11 \pm 0.84 \mathrm{~cm})$ (Figure 5). Therefore, the range from $42.14 \pm$ $1.08 \mathrm{~cm}$ to $86.57 \pm 1.08$ and $37.11 \pm 0.84 \mathrm{~cm}$ and $74.76 \pm 1.20 \mathrm{~cm}$ under non-stress and stress condition respectively. ANOVA results have indicated that the height of plant is found to be significant.

Though, plant height is basically a genetically controlled character, it is being influenced by environmental conditions and management practices and also a several reports indicating hat height will be reduced due to water stress (Evans, 1975; Rahman and Uddin, 2000). The present study revealed significant differences in plant height at all stages. Among the all genotypes, Pusa-362 $(86.430 \mathrm{~cm})$ recorded significantly higher plant height as compared to other genotypes and lower was noticed in Digvijay $(52.11 \mathrm{~cm})$ and ICC $867(37.11 \mathrm{~cm})$ in both the year respectively. The results are similar to those of Katiyar et al., (1977) and Rao and Kumar (2000) who also reported differences for plant height among different chickpea genotypes under water stress. The observations recorded in the present investigation were in agreement with the findings of Hussain et al., (2015).

\section{Primary branches per plant}

The data reveals that amongst the control and drought environment, non significantly highest number of primary branches plants ${ }^{-1}$ were recorded in control during both the years. With drought stress under terminal moisture stress there was a non significant and concomitant reduction in the number of primary branches plant $^{-1}$ during both the years. The detailed results evaluated under 
control condition as well as stress condition for both the years are represented in Table 3 and 4 respectively.

In Rabi season 2018-2019 among the chickpea genotypes, under non-stress condition maximum number of primary branches plant ${ }^{-1}$ was recorded in Pusa-362

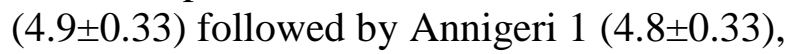
GNG-469 (4.5 \pm 0.57$)$ and ICC 4958 $(3.8 \pm 0.33)$ while minimum number of primary branches plant $^{-1}$ was recorded in shows in ICC $867(3.0 \pm 0.57)$, whereas under stress condition maximum number of primary branches plant ${ }^{-1}$ was recorded in GNG 469

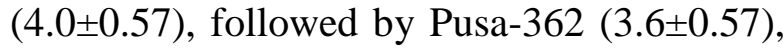
Annigeri $1 \quad(3.5 \pm 0.57)$ and ICC 4958 $(3.5 \pm 0.57)$ while minimum number of primary branches plant $^{-1}$ was recorded in recorded in Vishal (2.1 \pm 0.33$)$ (Figure 6). Maximum reduction percent was recorded in Vishal $(35.04 \%)$ and minimum was recorded in ICC $4958(8.70 \%)$ followed by ICC 867 $(8.89 \%)$ (Table 5). Therefore, the range from 3.0 to 4.9 and 2.1 to $4.0 \mathrm{~cm}$ under non-stress and stress condition, respectively.

But, in rabi season 2019-2020, data w.r.t. primary branches plant $^{-1}$ were having variation over the first year this variation w.r.t. number of primary branches plant $^{-1}$ in both the year, may be due to environmental effects. The results revealed that primary branches plant $^{-1}$ decreases nonsignificantly with water stress in all the ten varieties. Maximum percent reduction was recorded in Vishal $(27.00 \%)$ while the minimum percent reduction was observed in 1CC 4958 (10.48 $\%)$ and ICC 867 (11.76\%) (Table 6). Result further showed under non stress condition variety Pusa-362 (4.6 \pm 0.57$)$ recorded the maximum number of primary branches plant ${ }^{-1}$ closely followed by GNG-469 (4.5 \pm 0.5$)$; Digvijay (4.5 \pm 0.33$)$ and ICC 14778 (4.1 \pm 0.33$)$ (Figure 7) whereas the minimum number of primary branches plant $^{-1}$ was recorded in ICC $4958(2.8 \pm 0.57)$. While under water stress condition variety Pusa-362 (3.9 \pm 0.88$)$ showed the maximum number of primary branches plant $^{-1}$ closely followed by GNG-469 (3.8 \pm 0.72$)$, Digvijay $(3.7 \pm 0.44)$ and JG $11(3.5 \pm 0.88)$, and whereas the minimum number of primary branches plant $^{-1}$ was recorded in ICC 4958 (2.5 \pm 0.57$)$ (Table 4). Therefore, the range from 2.8 to 4.6 and 2.5 to 3.9 under non-stress and stress condition, respectively. ANOVA results have indicated that the number of primary branches plant $^{-1}$ is found to be nonsignificant.

Hussain et al., (2015) also reported the similar genotypic variation in number of branches plant $^{-1}$ was decline under the water stress at flowering and pod formation stage.

\section{Secondary branches per plant}

The data reveals that amongst the control and drought environment, significantly highest number of secondary branches plants ${ }^{-1}$ was recorded in control during both the years. With drought stress under terminal moisture stress there was a significant and concomitant reduction in the number of secondary branches plant ${ }^{-1}$ during both the years. The detailed results evaluated under control condition as well as stress condition for both the years are represented in Table 3 and 4 respectively.

In Rabi season 2018-2019 among the chickpea genotypes, under non-stress condition maximum number of secondary branches plant ${ }^{-1}$ was recorded in GNG-469 (17.50 \pm 0.57$)$ followed by Annigeri 1

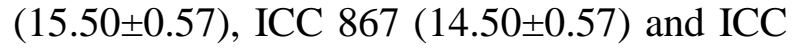
$14778(14.40 \pm 0.57)$ while minimum number of secondary branches plant ${ }^{-1}$ was recorded in Pusa-362 (8.33 \pm 0.57$)$, whereas under stress condition maximum number of secondary branches plant ${ }^{-1}$ was recorded in GNG 469 (14.50 \pm 0.57$)$, followed by DCP-92-3 
(12.66 \pm 0.88$)$, ICC $14778(12.60 \pm 0.57)$ and Annigeri 1; ICC $867 \quad(12.50 \pm 0.57)$ while minimum number of secondary branches plant $^{-1}$ was recorded in recorded in Pusa-362 (6.83 \pm 0.33 ) (Figure 8). Maximum reduction precent was recorded in Vishal $(26.96 \%)$ and minimum was recorded in ICC $4958(3.11 \%)$ and ICC 867 (6.90\%) (Table 5). Therefore, the range from 8.33 to 17.50 and 6.83 to 14.50 under non-stress and stress condition, respectively.

But, the Rabi season 2019-2020 data w.r.t. secondary branches plant $^{-1}$ were having variation over the first year this variation w.r.t. secondary branches plant ${ }^{-1}$, may be due to environmental effects. The results revealed that secondary branches plant $^{-1}$ decreases significantly with water stress in all the ten varieties. Result further showed under non stress condition variety Annigeri 1 $(16.3 \pm 0.88)$ recorded the maximum number of secondary branches plant ${ }^{-1}$ closely followed by GNG-469 (15.50 \pm 0.57$)$, DCP-92-3; ICC 14778 (14.00) and JG-11; ICC 867 (13.50 \pm 0.57 ) whereas the minimum secondary branches plant $^{-1}$ was recorded in Pusa-362 (8.40 \pm 0.57$)$. While under water stress condition variety GNG (13.83 \pm 0.88$)$ showed the maximum secondary branches plant ${ }^{-1}$ closely followed by Annigeri 1 (13.50 \pm 0.57 ), ICC 14778; DCP-92-3 (12.00 \pm 0.57$)$ and ICC 867 (11.50 \pm 0.33 ), and whereas, the minimum secondary branches plant ${ }^{-1}$ was recorded in Vishal $(6.16 \pm 0.72)$ (Figure 9). Maximum reduction percent was recorded in Vishal $(38.33 \%)$ and minimum was recorded in ICC $4958(5.80 \%)$ and ICC $867(9.88 \%)$ (Table 6). Therefore, the range from 8.40 to 16.33 and 6.16 to 13.83 under non-stress and stress condition, respectively. ANOVA results have indicated that the secondary branches plant ${ }^{-1}$ is found to be significant.

Table.1 Description of chickpea genotypes used under study

\begin{tabular}{|c|c|c|c|c|c|}
\hline $\begin{array}{l}\text { S. } \\
\text { No. }\end{array}$ & Genotypes & Parentage & Type & $\begin{array}{l}\text { Originating } \\
\text { center }\end{array}$ & $\begin{array}{l}\text { Drought } \\
\text { response }\end{array}$ \\
\hline 1. & Annigeri-1 & $\begin{array}{l}\text { Local selection from germplasm of } \\
\text { Karanataka }\end{array}$ & Desi & $\begin{array}{l}\text { MPKV, } \\
\text { Rahuri }\end{array}$ & $\begin{array}{l}\text { Tolerant, } \\
\text { adapted variety }\end{array}$ \\
\hline 2. & JG 11 & $\begin{array}{l}\text { [(Phule G5 x Narsinghpur bold) } \mathrm{x} \\
\text { ICCC37] }\end{array}$ & Desi & $\begin{array}{l}\text { MPKV, } \\
\text { Rahuri }\end{array}$ & Tolerant \\
\hline 3. & ICC 4958 & $\begin{array}{l}\text { GW } 5 / 7 \text {, a drought tolerant breeding } \\
\text { line from ICRISAT }\end{array}$ & Desi & $\begin{array}{l}\text { MPKV, } \\
\text { Rahuri }\end{array}$ & $\begin{array}{l}\text { Moderately } \\
\text { tolerant }\end{array}$ \\
\hline 4. & Digvijay & Phule G-91028 × Bheema & Desi & $\begin{array}{l}\text { MPKV, } \\
\text { Rahuri }\end{array}$ & Susceptible \\
\hline 5. & Vishal & K $850 \times$ ICCL 80074 & Desi & $\begin{array}{l}\text { MPKV, } \\
\text { Rahuri }\end{array}$ & Susceptible \\
\hline 6. & Pusa 362 & (BG $203 \times$ × 179) x BC 203 & Desi & $\begin{array}{l}\text { MPKV, } \\
\text { Rahuri }\end{array}$ & Susceptible \\
\hline 7. & DCP-92-3 & Selection from germplasm & Desi & IIPR, Kanpur & Susceptible \\
\hline 8. & GNG-469 & Derivative of Annegirix H75-35 & Desi & IIPR, Kanpur & Susceptible \\
\hline 9. & ICC 867 & Germplasm accessions & Desi & NBPGR & Highly tolerant \\
\hline 10. & ICC 14778 & Germplasm accessions & Desi & NBPGR & Highly tolerant \\
\hline
\end{tabular}


Table.2 Meteorological observations recorded during experimental period

\begin{tabular}{|c|c|c|c|c|c|c|}
\hline Week & $\begin{array}{l}\text { Temp } \\
\text { Max } \\
\left({ }^{\circ} \mathrm{C}\right)\end{array}$ & $\begin{array}{l}\text { Temp } \\
\text { Min } \\
\left({ }^{\circ} \mathrm{C}\right)\end{array}$ & $\begin{array}{c}\text { RH in } \\
\text { Morning } \\
(\%)\end{array}$ & $\begin{array}{c}\text { RH in } \\
\text { Evening } \\
(\%)\end{array}$ & $\begin{array}{c}\text { Rainfall } \\
\text { (mm) }\end{array}$ & BSS (h) \\
\hline week 45 & 26.0 & 10.7 & 96.8 & 69.0 & 0.0 & 1.4 \\
\hline week 46 & 27.7 & 13.1 & 94.8 & 52.7 & 0.0 & 4.6 \\
\hline week 47 & 25.1 & 6.7 & 57.9 & 28.1 & 0.0 & 7.7 \\
\hline week 48 & 24.9 & 6.1 & 95.7 & 28.4 & 0.0 & 7.4 \\
\hline week 49 & 24.3 & 7.9 & 87.7 & 28.6 & 0.0 & 5.3 \\
\hline week 50 & 20.0 & 8.4 & 90.9 & 52.1 & 10.0 & 3.5 \\
\hline week 51 & 23.3 & 7.9 & 96.3 & 38.1 & 0.0 & 4.5 \\
\hline week 52 & 23.0 & 6.4 & 94.3 & 41.9 & 0.0 & 5.7 \\
\hline week-1 & 15.4 & 5.9 & 91.9 & 68.7 & 0.0 & 2.5 \\
\hline week-2 & 21.9 & 6.4 & 96.0 & 35.9 & 0.0 & 6.6 \\
\hline week-3 & 23.6 & 6.9 & 91.7 & 40.4 & 0.0 & 6.8 \\
\hline week-4 & 19.7 & 6.6 & 94.3 & 55.3 & 0.0 & 4.6 \\
\hline week-5 & 24.7 & 7.8 & 94.4 & 32.9 & 0.0 & 7.6 \\
\hline week-6 & 23.9 & 6.6 & 90.6 & 26.6 & 0.0 & 6.4 \\
\hline week-7 & 23.3 & 9.0 & 87.9 & 45.4 & 3.6 & 7.4 \\
\hline week-8 & 30.1 & 11.1 & 87.6 & 34.9 & 0.2 & 7.1 \\
\hline week-9 & 31.4 & 13.4 & 76.4 & 31.6 & 0.0 & 7.5 \\
\hline week-10 & 31.3 & 12.3 & 83.7 & 31.3 & 0.0 & 8.9 \\
\hline week-11 & 32.3 & 14.3 & 84.4 & 30.3 & 0.0 & 8.3 \\
\hline week-12 & 33.7 & 15.9 & 81.0 & 37.7 & 0.0 & 8.0 \\
\hline week-13 & 35.3 & 16.7 & 80.7 & 33.6 & 0.0 & 8.4 \\
\hline week-14 & 37.6 & 20.7 & 78.0 & 44.1 & 0.0 & 7.2 \\
\hline week-15 & 33.1 & 19.1 & 84.4 & 48.6 & 24.0 & 8.4 \\
\hline week-16 & 39.7 & 22.0 & 65.0 & 19.4 & 0.0 & 8.9 \\
\hline week-17 & 40.7 & 23.3 & 61.4 & 17.6 & 0.0 & 9.8 \\
\hline Mean & 27.7 & 11.4 & 85.7 & 38.9 & 1.5 & 6.6 \\
\hline
\end{tabular}


Table.3 Morphological observations of chickpea genotypes under water stress (2018-2019) (n=03)

\begin{tabular}{|c|c|c|c|c|c|c|c|c|c|c|c|c|c|c|}
\hline \multirow{2}{*}{$\begin{array}{l}\text { Character } \rightarrow \\
\text { Genotypes } \downarrow\end{array}$} & \multicolumn{2}{|c|}{ DTF $50 \%$} & \multicolumn{2}{|c|}{ PH } & \multicolumn{2}{|c|}{ NP } & \multicolumn{2}{|c|}{ NPB } & \multicolumn{2}{|c|}{ NSB } & \multicolumn{2}{|c|}{ TW } & \multicolumn{2}{|c|}{ SY } \\
\hline & NS & $S$ & NS & $\mathrm{S}$ & NS & $S$ & NS & $S$ & NS & S & NS & $S$ & NS & $S$ \\
\hline Annigeri 1 & $47.66 \pm 1.15$ & $44.33 \pm 1.85$ & $74.78 \pm 1.23$ & $69.41 \pm 0.75$ & $43.00 \pm 1.15$ & $36.33 \pm 0.88$ & $4.8 \pm 0.33$ & $3.5 \pm 0.57$ & $15.5 \pm 0.57$ & $12.5 \pm 0.57$ & $25.81 \pm 1.61$ & $19.85 \pm 0.87$ & $17.30 \pm 0.09$ & $15.19 \pm 0.1$ \\
\hline JG 11 & $63.00 \pm 1.15$ & $58.33 \pm 0.88$ & $77.06 \pm 1.12$ & $63.47 \pm 1.55$ & $51.00 \pm 1.15$ & $44.67 \pm 2.40$ & $3.1 \pm 0.57$ & $2.8 \pm 0.57$ & $14.5 \pm 0.57$ & $11.5 \pm 0.57$ & $23.50 \pm .017$ & $20.38 \pm 0.13$ & $21.36 \pm 0.25$ & $17.92 \pm 0.38$ \\
\hline ICC 4958 & $47.33 \pm 1.45$ & $45.33 \pm 2.02$ & $60.86 \pm 1.29$ & $56.98 \pm 1.06$ & $35.00 \pm 1.73$ & $31.33 \pm 0.66$ & $3.8 \pm 0.33$ & $3.5 \pm 0.57$ & $12.6 \pm 0.57$ & $12.2 \pm 0.59$ & $30.10 \pm 0.20$ & $27.95 \pm 0.22$ & $20.48 \pm 0.14$ & $18.85 \pm 0.03$ \\
\hline Digvijay & $57.66 \pm 1.15$ & $52.66 \pm 0.88$ & $52.11 \pm 0.73$ & $43.60 \pm 0.81$ & $79.66 \pm 0.88$ & $64.00 \pm 1.52$ & $3.5 \pm 0.57$ & $2.8 \pm 0.33$ & $12.6 \pm 0.88$ & $10.6 \pm 0.88$ & $24.73 \pm 0.12$ & $22.83 \pm 0.38$ & $17.39 \pm 0.13$ & $14.52 \pm 0.16$ \\
\hline Vishal & $49.00 \pm 1.15$ & $43.33 \pm 1.76$ & $58.24 \pm 1.16$ & $42.79 \pm 1.20$ & $44.67 \pm 1.45$ & $28.33 \pm 0.88$ & $3.3 \pm 0.57$ & $2.1 \pm 0.33$ & $11.5 \pm 0.57$ & $8.4 \pm 0.57$ & $27.60 \pm 0.20$ & $19.98 \pm 0.09$ & $18.02 \pm 0.16$ & $14.54 \pm 0.19$ \\
\hline Pusa-362 & $70.00 \pm 0.88$ & $65.00 \pm 1.52$ & $86.43 \pm 1.30$ & $71.91 \pm 1.48$ & $74.66 \pm 0.66$ & $65.33 \pm 1.45$ & $4.9 \pm 0.33$ & $3.6 \pm 0.57$ & $8.33 \pm 0.57$ & $6.8 \pm 0.33$ & $20.39 \pm 0.03$ & $18.79 \pm 0.09$ & $20.33 \pm 0.26$ & $17.49 \pm 0.25$ \\
\hline DCP-92-3 & $71.66 \pm 1.15$ & $66.00 \pm 1.52$ & $71.00 \pm 1.07$ & $62.66 \pm 1.62$ & $48.00 \pm 0.57$ & $41.66 \pm 0.88$ & $3.6 \pm 0.66$ & $3.0 \pm 0.57$ & $15 \pm 0.57$ & $12.6 \pm 0.88$ & $22.28 \pm 0.02$ & $20.36 \pm 0.23$ & $19.25 \pm 0.55$ & $16.64 \pm 0.18$ \\
\hline GNG-469 & $76.33 \pm 2.08$ & $71.33 \pm 0.66$ & $84.06 \pm 1.28$ & $75.46 \pm 0.52$ & $78.66 \pm 0.33$ & $70.00 \pm 1.52$ & $4.5 \pm 0.57$ & $4 \pm 0.57$ & $17.5 \pm 0.57$ & $14.5 \pm 0.57$ & $26.24 \pm 0.29$ & $23.76 \pm 1.02$ & $18.13 \pm 0.32$ & $15.24 \pm 0.12$ \\
\hline ICC 867 & $52.00 \pm 0.57$ & $49.66 \pm 0.88$ & $57.67 \pm 0.53$ & $52.90 \pm 1.29$ & $73.33 \pm 2.02$ & $65.33 \pm 1.45$ & $3.0 \pm 0.57$ & $2.7 \pm 0.33$ & $14.5 \pm 0.57$ & $13.5 \pm 0.57$ & $14.40 \pm 0.12$ & $13.31 \pm 0.03$ & $17.12 \pm 0.27$ & $15.48 \pm 0.25$ \\
\hline ICC 14778 & $57.66 \pm 1.15$ & $51.66 \pm 0.88$ & $69.74 \pm 1.36$ & $62.34 \pm 1.18$ & $40.00 \pm 2.08$ & $33.66 \pm 0.88$ & $3.5 \pm 0.57$ & $2.6 \pm 0.33$ & $14.4 \pm 0.57$ & $12.6 \pm 0.57$ & $23.60 \pm 0.03$ & $21.67 \pm 0.09$ & $25.36 \pm 0.15$ & $21.31 \pm 0.18$ \\
\hline C.D. & 3.557 & 4.083 & 3.389 & 3.569 & 3.973 & 4.01 & N/A & N/A & 1.826 & 1.883 & 1.619 & 1.245 & 0.796 & 0.616 \\
\hline SE(m) & 1.197 & 1.374 & 1.141 & 1.201 & 1.337 & 1.35 & 0.527 & 0.494 & 0.615 & 0.634 & 0.545 & 0.419 & 0.268 & 0.208 \\
\hline SE(d) & 1.693 & 1.944 & 1.613 & 1.699 & 1.892 & 1.909 & 0.745 & 0.699 & 0.869 & 0.896 & 0.771 & 0.593 & 0.379 & 0.293 \\
\hline C.V. & 3.511 & 4.347 & 2.855 & 3.462 & 4.079 & 4.864 & 23.862 & 27.779 & 7.796 & 9.511 & 3.955 & 3.474 & 2.384 & 2.15 \\
\hline
\end{tabular}

Table.4 Morphological observations of chickpea genotypes under water stress (2019-2020) (n=03)

\begin{tabular}{|c|c|c|c|c|c|c|c|c|c|c|c|c|c|c|}
\hline \multirow{2}{*}{$\begin{array}{l}\text { Character } \rightarrow \\
\text { Genotypes } \downarrow\end{array}$} & \multicolumn{2}{|c|}{ DTF $50 \%$} & \multicolumn{2}{|c|}{ PH } & \multicolumn{2}{|c|}{ NPB } & \multicolumn{2}{|c|}{ NSB } & \multicolumn{2}{|c|}{ NPP } & \multicolumn{2}{|c|}{ TW } & \multicolumn{2}{|c|}{ SY } \\
\hline & NS & S & NS & S & NS & S & NS & S & NS & S & NS & S & NS & S \\
\hline Annigeri 1 & $50.33 \pm 1.15$ & $42.00 \pm 1.15$ & $64.73 \pm 2.32$ & $53.02 \pm 1.42$ & $3.5 \pm 0.57$ & $2.6 \pm 0.44$ & $16.3 \pm 0.88$ & $13.5 \pm 0.57$ & $41.33 \pm 0.88$ & $37.33 \pm 1.45$ & $29.68 \pm 0.05$ & $25.49 \pm 0.25$ & $19.46 \pm 0.27$ & $17.22 \pm 0.05$ \\
\hline JG 11 & $61.33 \pm 0.88$ & $57.33 \pm 2.33$ & $69.87 \pm 1.24$ & $63.54 \pm 0.83$ & $4.1 \pm 0.57$ & $3.4 \pm 0.88$ & $13.5 \pm 0.57$ & $10.4 \pm 0.49$ & $50.00 \pm 1.73$ & $45.33 \pm 1.45$ & $22.4 \pm 0.22$ & $19.98 \pm 0.09$ & $24.30 \pm 0.08$ & $19.49 \pm 0.11$ \\
\hline ICC 4958 & $44.66 \pm 1.45$ & $42.66 \pm 1.45$ & $75.73 \pm 0.13$ & $72.98 \pm 1.44$ & $2.8 \pm 0.57$ & $2.5 \pm 0.57$ & $11.5 \pm 0.57$ & $10.8 \pm 0.16$ & $35.66 \pm 1.20$ & $34.00 \pm 0.57$ & $27.60 \pm 0.38$ & $26.20 \pm 0.52$ & $19.22 \pm 0.02$ & $17.66 \pm 0.08$ \\
\hline Digvijay & $54.66 \pm 1.45$ & $48.66 \pm 2.33$ & $57.94 \pm 1.20$ & $51.06 \pm 0.94$ & $4.5 \pm 0.33$ & $3.6 \pm 0.44$ & $12 \pm 0.57$ & $10 \pm 0.57$ & $73.33 \pm 0.66$ & $62.00 \pm 1.52$ & $22.31 \pm 0.18$ & $17.27 \pm 0.10$ & $16.39 \pm 0.07$ & $13.16 \pm 0.07$ \\
\hline Vishal & $50.33 \pm 1.45$ & $39.33 \pm 1.76$ & $48.93 \pm 1.26$ & $37.44 \pm 1.04$ & $3.8 \pm 0.88$ & $2.8 \pm 0.57$ & $10 \pm 0.57$ & $6.1 \pm 0.72$ & $42.33 \pm 1.45$ & $30.33 \pm 1.45$ & $27.07 \pm 0.46$ & $20.15 \pm 0.03$ & $17.27 \pm 0.10$ & $13.74 \pm 0.25$ \\
\hline Pusa-362 & $71.33 \pm 0.88$ & $66.00 \pm 1.52$ & $80.93 \pm 1.07$ & $70.43 \pm 1.03$ & $4.6 \pm 0.57$ & $3.9 \pm 0.88$ & $8.4 \pm 0.57$ & $7.3 \pm 0.60$ & $77.66 \pm 1.45$ & $70.00 \pm 1.73$ & $19.85 \pm 0.08$ & $16.39 \pm 0.07$ & $19.48 \pm 0.11$ & $17.48 \pm 1.68$ \\
\hline DCP-92-3 & $68.33 \pm 0.88$ & $64.33 \pm 2.33$ & $69.07 \pm 0.68$ & $62.21 \pm 1.25$ & $3.6 \pm 0.88$ & $3.0 \pm 0.57$ & $14 \pm 0.57$ & $12 \pm 0.57$ & $47.33 \pm 0.88$ & $43.00 \pm 1.73$ & $21.16 \pm 0.08$ & $17.48 \pm 1.16$ & $18.89 \pm 0.26$ & $16.46 \pm 0.10$ \\
\hline GNG-469 & $74.66 \pm 0.88$ & $65.00 \pm 1.73$ & $85.90 \pm 1.08$ & $74.76 \pm 1.20$ & $4.5 \pm 0.57$ & $3.8 \pm 0.72$ & $15.5 \pm 0.57$ & $13.8 \pm 0.88$ & $70.33 \pm 0.88$ & $64.00 \pm 1.73$ & $25.57 \pm 0.09$ & $23.37 \pm 0.17$ & $17.28 \pm 0.08$ & $14.34 \pm 0.06$ \\
\hline ICC 867 & $53.00 \pm 1$ & $50.00 \pm 0.57$ & $42.14 \pm 1.08$ & $37.11 \pm 0.84$ & $3.5 \pm 0.57$ & $3.1 \pm 0.46$ & $13.5 \pm 0.57$ & $12.1 \pm 0.33$ & $75.33 \pm 1.45$ & $71.66 \pm 0.88$ & $16.41 \pm 0.2$ & $15.01 \pm 0.31$ & $17.37 \pm 0.14$ & $15.87 \pm 0.02$ \\
\hline ICC 14778 & $56.00 \pm 0.57$ & $52.33 \pm 1.20$ & $65.86 \pm 1.71$ & $59.18 \pm 1.03$ & $4.1 \pm 0.33$ & $3.1 \pm 0.33$ & $14.0 \pm 0.57$ & $12.0 \pm 0.8$ & $37.00 \pm 1.52$ & $32.66 \pm 0.66$ & $17.28 \pm 0.08$ & $13.43 \pm 0.04$ & $20.15 \pm 0.39$ & $18.35 \pm 0.18$ \\
\hline C.D. & 3.269 & 5.146 & 3.865 & 3.342 & N/A & N/A & 1.826 & 1.663 & 3.745 & 4.119 & 0.677 & 1.283 & 0.429 & 1.152 \\
\hline SE(m) & 1.101 & 1.732 & 1.301 & 1.125 & 0.615 & 0.617 & 0.615 & 0.56 & 1.261 & 1.386 & 0.228 & 0.432 & 0.144 & 0.388 \\
\hline $\operatorname{SE}(d)$ & 1.556 & 2.449 & 1.84 & 1.591 & 0.869 & 0.872 & 0.869 & 0.791 & 1.783 & 1.961 & 0.322 & 0.611 & 0.204 & 0.548 \\
\hline C.V. & 3.262 & 5.685 & 3.408 & 3.349 & 27.086 & 33.136 & 8.27 & 8.956 & 3.967 & 4.897 & 1.722 & 3.841 & 1.317 & 4.1 \\
\hline
\end{tabular}


Table.5 Percent changes in Morphological observations in Chickpea genotypes under water stress (2018-2019)

\begin{tabular}{|c|c|c|c|c|c|c|c|}
\hline $\begin{array}{l}\text { Character } \rightarrow \\
\text { Genotypes } \downarrow\end{array}$ & $\begin{array}{c}\text { DTF } \\
\text { 50\% }\end{array}$ & PH & NPP & NPB & NSB & TW & SY \\
\hline Annigeri 1 & 5.67 & 7.19 & 15.50 & 27.59 & 19.35 & 23.09 & 12.21 \\
\hline JG 11 & 7.41 & 18.19 & 12.42 & 10.44 & 20.69 & 13.28 & 16.09 \\
\hline ICC 4958 & 4.90 & 6.38 & 10.48 & 8.70 & 3.11 & 8.75 & 7.93 \\
\hline Digvijay & 7.60 & 16.32 & 19.67 & 19.05 & 15.79 & 6.47 & 16.48 \\
\hline Vishal & 12.75 & 26.54 & 36.57 & 35.04 & 26.96 & 27.61 & 19.31 \\
\hline Pusa-362 & 7.14 & 16.80 & 12.50 & 27.03 & 17.97 & 7.54 & 14.00 \\
\hline DCP-92-3 & 7.04 & 11.74 & 13.19 & 18.18 & 15.56 & 8.59 & 13.54 \\
\hline GNG-469 & 6.14 & 10.23 & 11.02 & 11.11 & 17.14 & 9.44 & 15.96 \\
\hline ICC 867 & 5.10 & 8.27 & 10.91 & 8.89 & 6.90 & 8.01 & 9.61 \\
\hline ICC 14778 & 9.36 & 10.61 & 15.83 & 23.81 & 12.50 & 8.20 & 15.96 \\
\hline
\end{tabular}

Table.6 Percent changes in morphological observations in Chickpea genotypes under water stress (2019-2020)

\begin{tabular}{|l|c|c|c|c|c|c|c|}
\hline $\begin{array}{l}\text { Character } \rightarrow \\
\text { Genotypes } \downarrow\end{array}$ & $\begin{array}{l}\text { DTF } \\
\mathbf{5 0 \%}\end{array}$ & PH & NPP & NPB & NSB & TW & SY \\
\hline Annigeri 1 & 16.00 & 11.71 & 9.68 & 23.81 & 17.35 & 14.12 & 11.51 \\
\hline JG 11 & 6.52 & 6.33 & 9.33 & 15.95 & 22.96 & 10.80 & 19.79 \\
\hline ICC 4958 & 4.48 & 2.74 & 4.67 & 10.48 & 5.80 & 5.10 & 8.10 \\
\hline Digvijay & 10.98 & 6.88 & 15.45 & 18.52 & 16.67 & 22.60 & 19.72 \\
\hline Vishal & 21.85 & 11.49 & 28.35 & 27.00 & 38.33 & 25.55 & 20.46 \\
\hline Pusa-362 & 7.48 & 10.50 & 9.87 & 14.49 & 12.70 & 17.41 & 10.28 \\
\hline DCP-92-3 & 5.85 & 6.86 & 9.15 & 18.18 & 14.29 & 17.39 & 12.86 \\
\hline GNG-469 & 12.95 & 11.14 & 9.00 & 14.81 & 10.75 & 8.61 & 17.01 \\
\hline ICC 867 & 5.66 & 5.02 & 4.87 & 11.76 & 9.88 & 8.53 & 8.60 \\
\hline ICC 14778 & 6.55 & 6.67 & 11.71 & 24.00 & 14.29 & 22.31 & 8.95 \\
\hline
\end{tabular}

Fig.1 Morphological Parameters Measurement

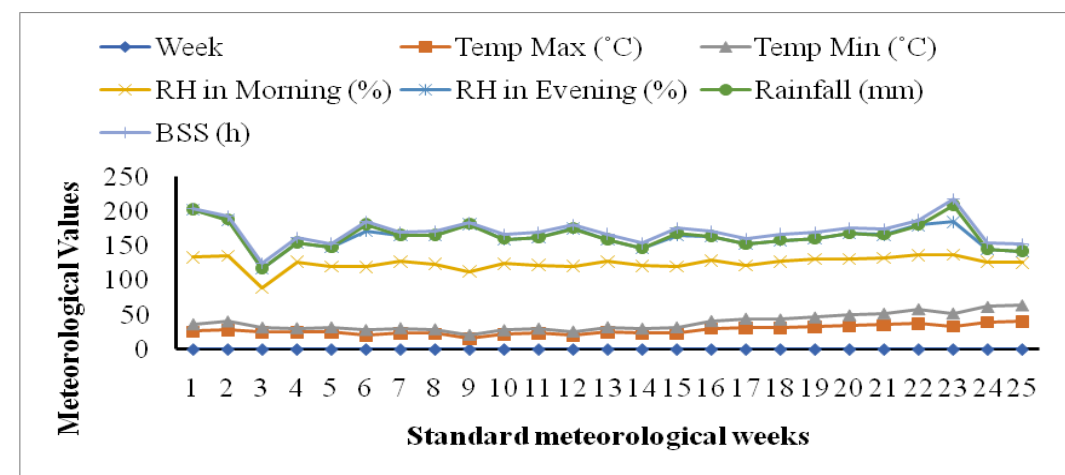


Fig.2 Days to 50 per cent flowering plants ${ }^{-1}$ of chickpea genotypes under two levels of water stress, season 2018. Bars represent mean Days to 50 per cent flowering of three biological replication and error bars indicated the standard error $( \pm \mathrm{SE})$

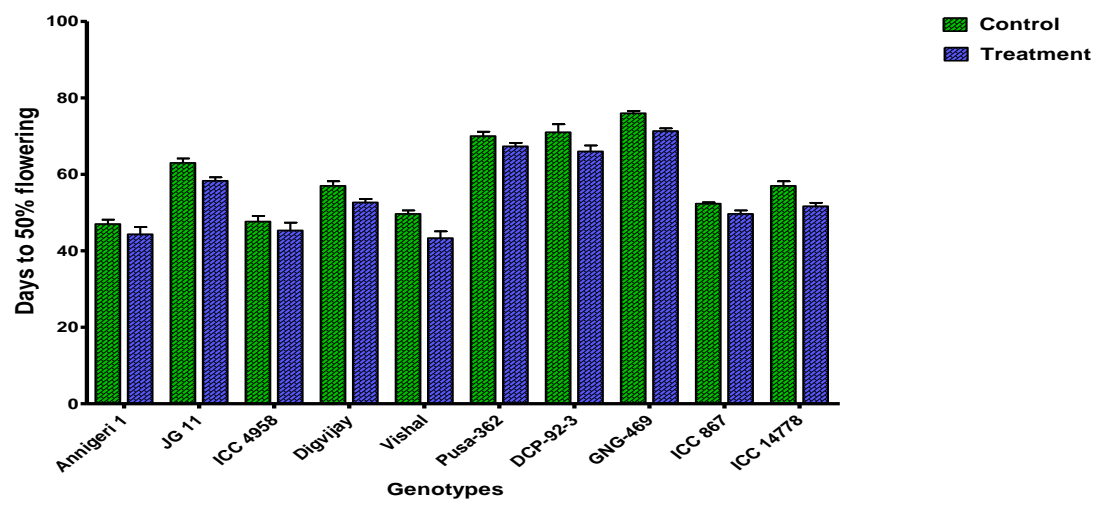

Fig.3 Days to 50 per cent flowering plants ${ }^{-1}$ of chickpea genotypes under two levels of water stress, season 2019. Bars represent mean Days to 50 per cent flowering of three biological replication and error bars indicated the standard error $( \pm \mathrm{SE})$

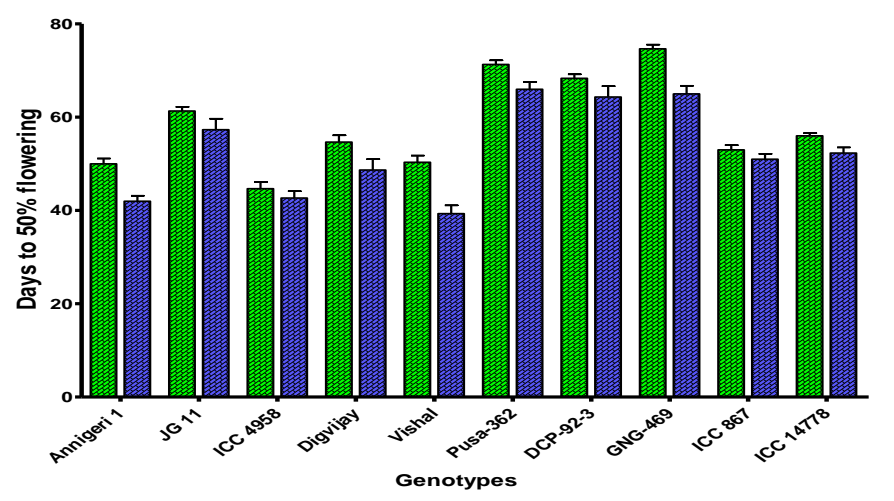

圂 Control

图 Treatment

Fig.4 Plant height plants ${ }^{-1}$ of chickpea genotypes under two levels of water stress, season 2018. Bars represent mean Plant height of three biological replication and error bars indicated the standard error $( \pm \mathrm{SE})$

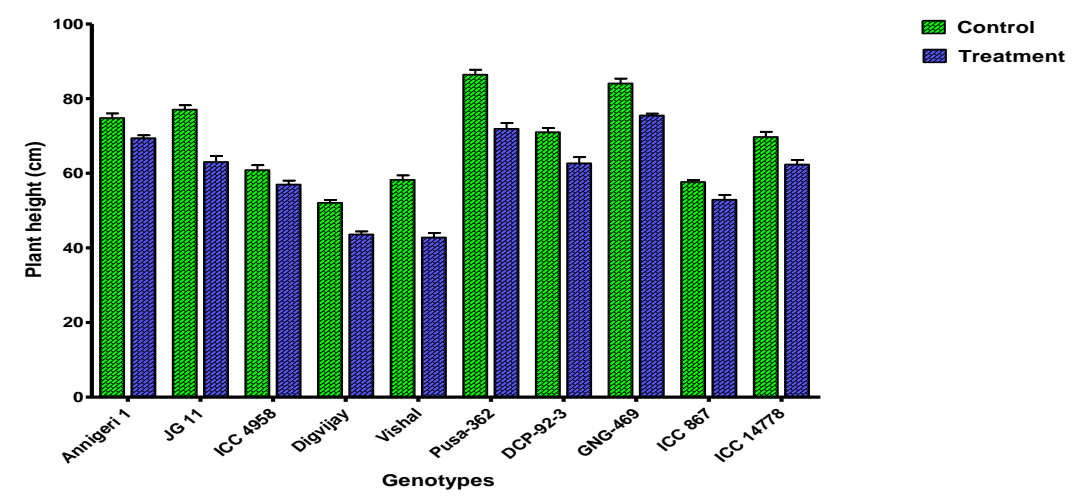


Fig.5 Plant height plants $^{-1}$ of chickpea genotypes under two levels of water stress, season 2019. Bars represent mean Plant height of three biological replication and error bars indicated the standard error $( \pm \mathrm{SE})$

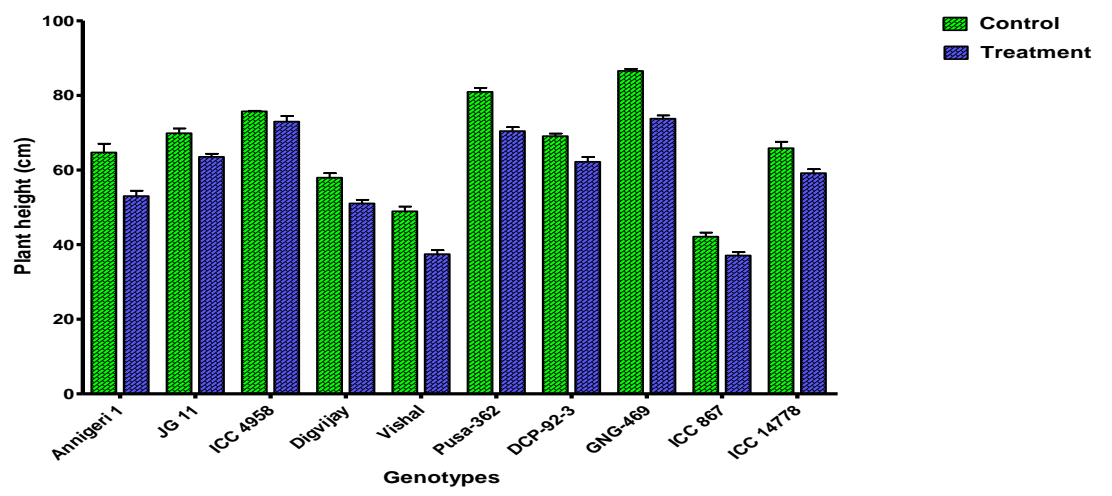

Fig.6 Primary branchesplants ${ }^{-1}$ of chickpea genotypes under two levels of water stress, season 2018. Bars represent mean primary branches of three biological replication and error bars indicated the standard error $( \pm \mathrm{SE})$

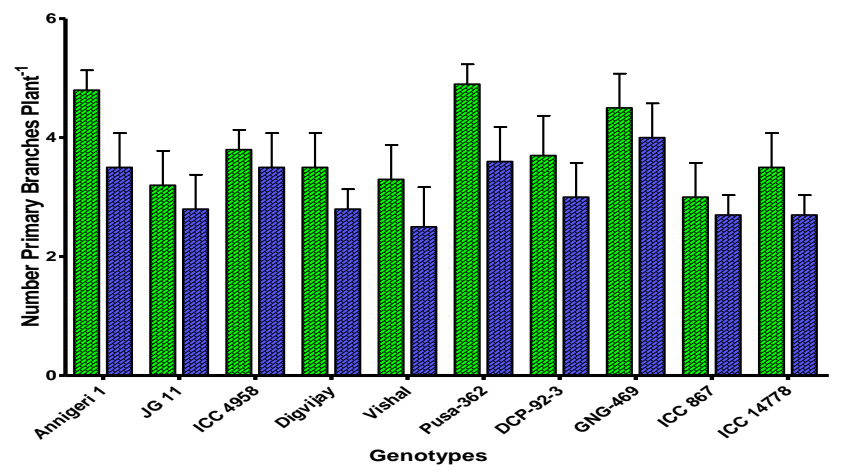

Fig.7 Primary branchesplants ${ }^{-1}$ of chickpea genotypes under two levels of water stress, season 2019. Bars represent mean primary branches of three biological replication and error bars indicated the standard error $( \pm \mathrm{SE})$

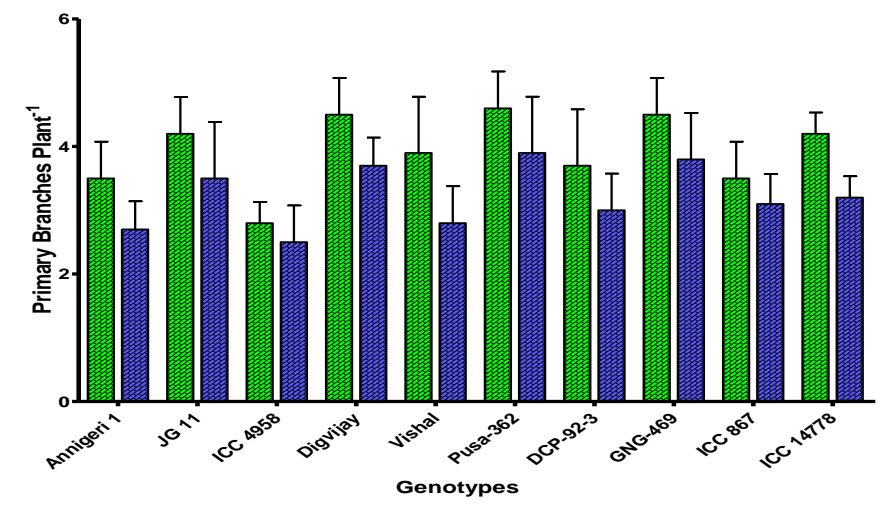

Control 
Fig.8 Secondary branchesplants ${ }^{-1}$ of chickpea genotypes under two levels of water stress, season 2018. Bars represent mean secondary branches of three biological replication and error bars indicated the standard error $( \pm \mathrm{SE})$

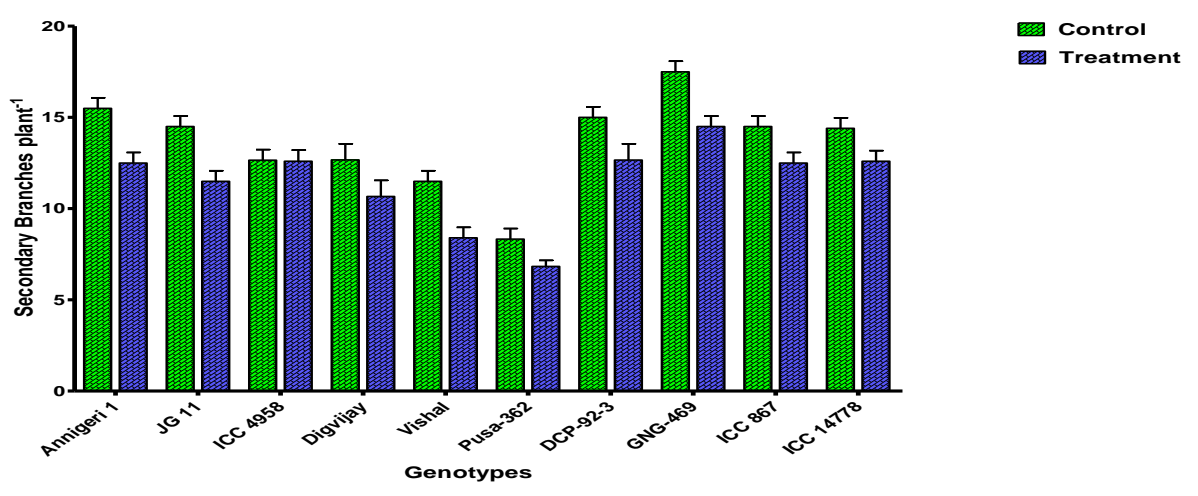

Fig.9 Secondary branchesplants ${ }^{-1}$ of chickpea genotypes under two levels of water stress, season 2019. Bars represent mean secondary branches of three biological replication and error bars indicated the standard error $( \pm \mathrm{SE})$

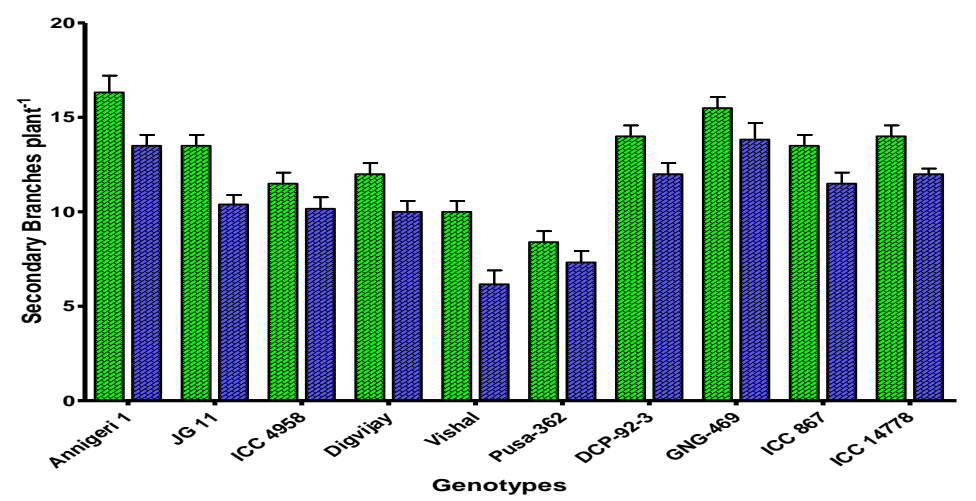

Control

Treatment

Fig.10 Podsplant ${ }^{-1}$ of chickpea genotypes under two levels of water stress, season 2018. Bars represent mean Podsplant ${ }^{-1}$ of three biological replication and error bars indicated the standard error $( \pm \mathrm{SE})$

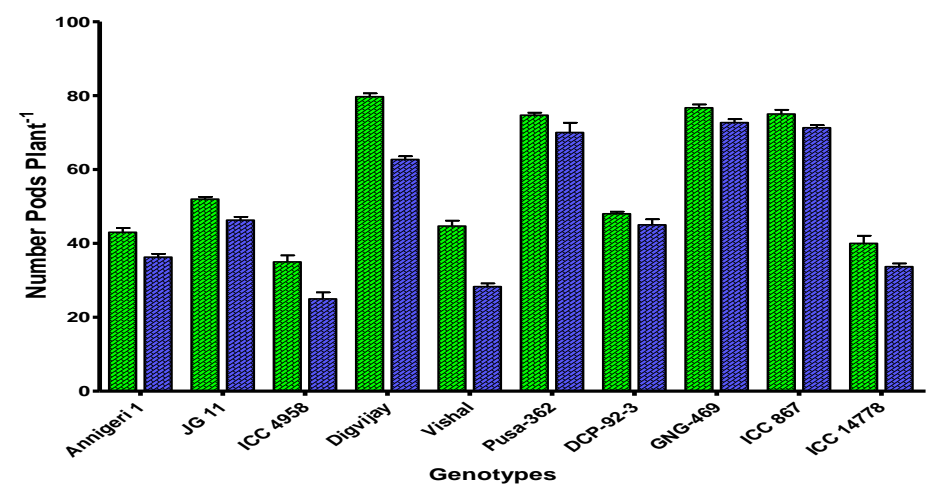

Control

Treatment 
Fig.11 Podsplant ${ }^{-1}$ of chickpea genotypes under two levels of water stress, season 2019. Bars represent mean Podsplant ${ }^{-1}$ of three biological replication and error bars indicated the standard error $( \pm \mathrm{SE})$

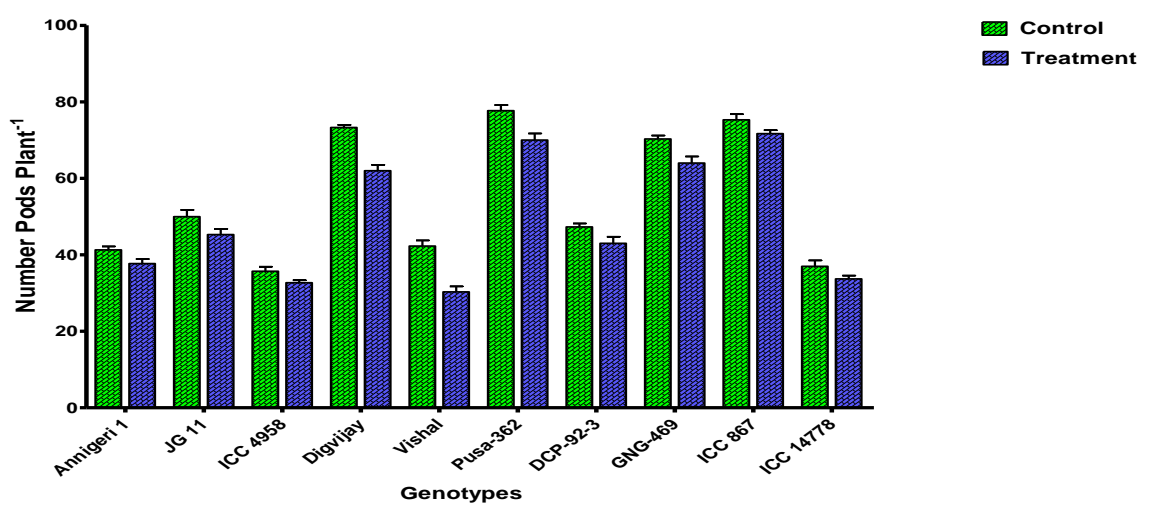

Fig.12 Test weight of chickpea genotypes under two levels of water stress, season 2018. Bars represent mean Test weight of three biological replication and error bars indicated the standard error $( \pm \mathrm{SE})$

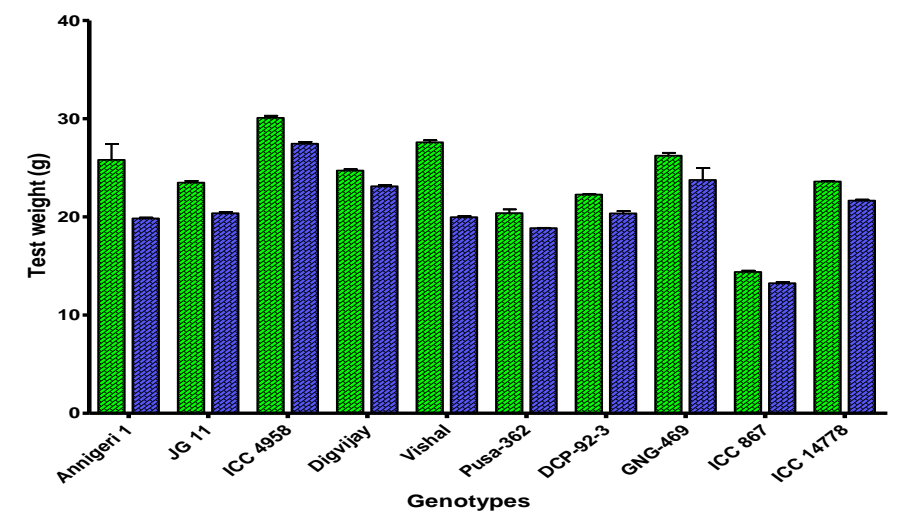

Control
Treatment

Fig.13 Test weight of chickpea genotypes under two levels of water stress, season 2019. Bars represent mean Test weight of three biological replication and error bars indicated the standard error $( \pm \mathrm{SE})$

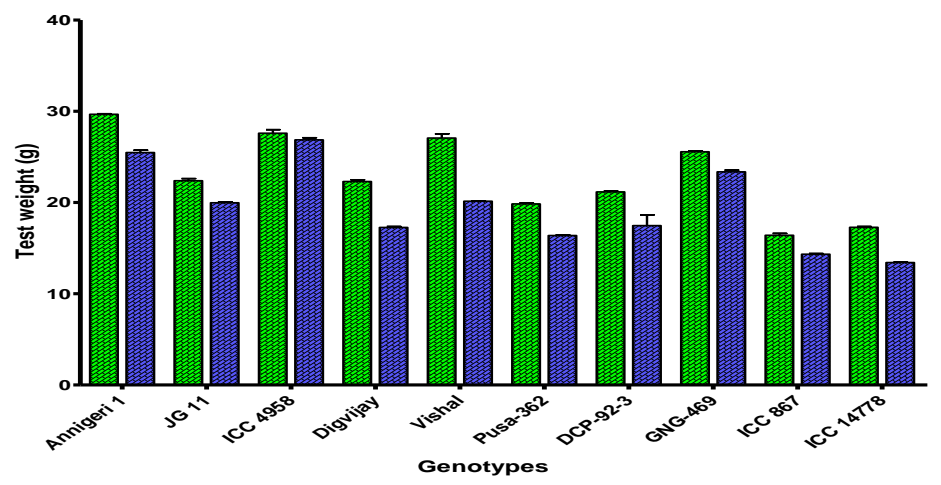

Control

Treatment 
Fig.14 Yield plant ${ }^{-1}$ of chickpea genotypes under two levels of water stress rabi season 2018. Bars represent mean Yield plant ${ }^{-1}$ of three biological replication and error bars indicated the standard error $( \pm$ SE $)$

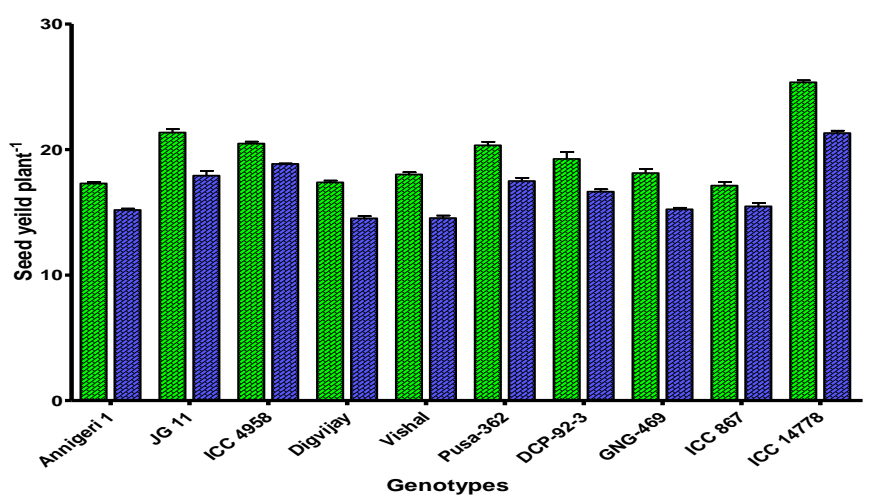

Control

Treatment

Fig.15 Yield plant ${ }^{-1}$ of chickpea genotypes under two levels of water stress rabi season 2019. Bars represent mean Yield plant ${ }^{-1}$ of three biological replication and error bars indicated the standard error $( \pm \mathrm{SE})$

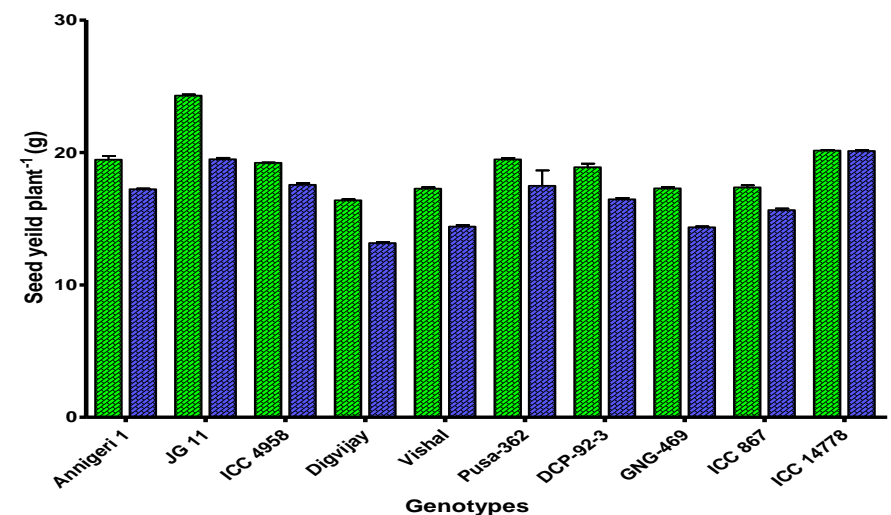

Control

国 Treatment

In the present study, significant difference among the genotypes in response to number of secondary branches plant ${ }^{-1}$ presented in Table 3 and 4 reveals that the rainfed environment led to a drastic decline in the number of secondary branches plant ${ }^{-1}$ in comparison to the control and water stress treatment. This might be due to the shortage of assimilates required for increased branching due to lower leaf area under moisture deficit environment. Similar results were reported by Rahman and Uddin (2000). Hussain et al., (2015) also reported that the secondary branches plant ${ }^{-1}$ also decline water stress treatment as compare to the control.

\section{Number of pods per plant}

The data reveals that amongst the control and drought environment, significantly highest number of pods plants ${ }^{-1}$ was recorded in control during both the years. A reference to the data shown in Table 3 and 4 illustrates the impact of water stress on the number of Pods plant $^{-1}$ in ten genotypes of chickpea at harvest time. Rabi season 2018-2019 data shows that, with water stress, the amount of Pods plant ${ }^{-1}$ decreased significantly in all ten genotypes. The minimum decrease was reported in ICC $4958(10.48 \%)$ followed by ICC 867 $(10.91 \%)$ and the maximum decrease in 
Vishal (36.57 \%) (Table 5). The Digvijay (79.66 \pm 0.88$)$ variety shows the maximum number of seeds per pod under environments of non-stress followed by GNG-469 (78.66 \pm 0.33$)$, Pusa-362 (74.66 \pm 0.66$)$ and ICC $867(73.33 \pm 2.02)$ whereas the minimum number of Pods plant ${ }^{-1}$ was recorded in ICC 4958 (35 \pm 1.73$)$. While under water stress condition variety GNG $(70 \pm 1.52)$ showed the maximum number of Pods plant ${ }^{-1}$ closely followed by ICC 867; Pusa-362 (65.33 \pm 1.45$)$, and Digvijay $(64 \pm 1.52)$ and whereas, the minimum number of Pods plant ${ }^{-1}$ was recorded in Vishal $(28.33 \pm 0.88)$ (Figure 10). Therefore, the range from 35.00 to 79.66 and 28.33 to 70.00 under non-stress and stress condition, respectively. ANOVA results have indicated that the number of Pods plant ${ }^{-1}$ is found to be significant.

In rabi season 2019-2020 the data shows that, with water stress, the amount of Pods plant ${ }^{-1}$ decreased significantly in all ten genotypes. The minimum decrease was reported in ICC $4958(4.67 \%)$ followed by ICC $867(4.87 \%)$ and the maximum decrease in Vishal $(28.35 \%)$ (Table 4). The ICC 867 $(71.66 \pm 0.88)$ variety shows the maximum number of seeds per pod under environments of water stress followed by Pusa-362 (70 \pm 1.73$)$, and GNG-469 $(64 \pm 1.73)$ whereas the minimum number of Pods plant ${ }^{-1}$ was recorded in ICC 4958 (30.33 \pm 1.45$)$. While under non water stress condition variety Pusa362 (77.66 \pm 1.45$)$ showed the maximum number of Pods plant ${ }^{-1}$ closely followed by ICC 867 (75.33 \pm 1.45$)$, Digvijay (73.33 \pm 0.66$)$ and GNG-469 (70.33 \pm 0.88$)$ whereas, the minimum number of Pods plant ${ }^{-1}$ was recorded in ICC $4958(35.66 \pm 1.20)$ (Figure 11). Therefore, the range from 30.00 to 71.66 and 35.66 to 77.66 under stress and non-stress condition, respectively. ANOVA results have indicated that the number of Pods plant ${ }^{-1}$ is found to be significant.
In the present study the number of pods per plant in different genotypes ranged from (35.00 to 79.66 and 28.33 to 70.00 ) and (30.00 to 71.66 and 35.66 to 77.66 ) in both year under control and water stress, respectively. It was observed that the genotypes Digvijay and ICC 867 produced significantly higher number of pods and the genotype Vishal and ICC 4958 produced significantly lowest number of pods per plant due to significantly less number of seeds per pod, number of seeds per plant and 100 grain weight $(\mathrm{g})$. Similar results were obtained by Hussain et al., (2015) and Tripathi et al., (1995). Reddy and Ahlawat (1998) also reported the variation in chickpea in relation to the number of pods per plant under control and stress condition.

\section{Test weight (g)}

Data presented in table 3 and Table 4 showed that the test weight differ significantly with respect to control and treatment during both years. The test weight decreases significantly under water stress condition.

Results further showed that the test weight also differed significantly under different varieties of chickpea. Under non stress condition variety ICC $4958(30.1 \pm 0.20 \mathrm{~g})$ shows highest test weight closely followed by Vishal (27.6 $\pm 0.20 \mathrm{~g})$, GNG-469 (26.24 \pm 0.29 $\mathrm{g})$ and Annigeri $1(25.81 \pm 1.61 \mathrm{~g})$ whereas the minimum test weight was recorded in ICC

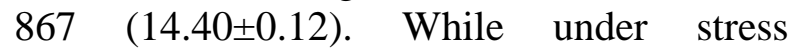
condition variety ICC $4958(27.95 \pm 0.22 \mathrm{~g})$ shows highest test weight closely followed by GNG-469 (23.76 \pm 1.02 g), Digvijay $(22.83 \pm 0.38 \mathrm{~g})$ and ICC $14778(21.67 \pm 0.09 \mathrm{~g})$ whereas the minimum test weight was recorded in ICC $867(13.31 \pm 0.03)$ (Figure 12). The minimum reduction was recorded in ICC $4958(8.75 \%)$ followed by ICC 867 $(8.01 \%)$ and maximum reduction found in Vishal $(27.61 \%)$ (Table 5). Therefore, the 
range from 14.40 to 30.1 and 13.31 to 27.95 under non-stress and stress condition, respectively.

In rabi season 2019-2020 results further showed that the test weight also differed significantly under different varieties of chickpea. Under non stress condition variety Annigeri 1 (29.68 $\pm 0.05 \mathrm{~g})$ shows highest test weight closely followed by ICC 4958 $(27.60 \pm 0.38 \mathrm{~g})$, Vishal $(27.07 \pm 0.46 \mathrm{~g})$ and GNG-469 (25.57 $\pm 0.09 \mathrm{~g})$ whereas the minimum test weight was recorded in ICC $867(16.41 \pm 0.2)$.

While under stress condition variety ICC $4958(26.2 \pm 0.52 \mathrm{~g})$ shows highest test weight closely followed by Annigeri 1 (25.49 \pm 0.25 g), GNG-469 (23.37 \pm 0.17 g)and Vishal $(20.15 \pm 0.03 \mathrm{~g})$ whereas the minimum test weight was recorded in ICC 14778 (13.43 \pm 0.04$)$ (Figure 13). The minimum reduction was recorded in ICC $4958(5.10 \%)$ followed by ICC 867 (8.53\%) and maximum reduction found in Vishal (25.55\%) (Table 6). Therefore, the range from 16.41 to 29.68 and 13.43 to 26.02 under non-stress and stress condition, respectively.

100 seed weight in chickpea in different genotypes were showing statistically significant indicating the considerable variations. 100 seed weight ranged from 14.40 to 30.1 and 13.31 to 27.95 under nonstress and stress condition, respectively. It was observed that the genotypes ICC 4958 and Annigeri 1 produced significantly higher 100 seed weight and the genotype ICC 867 and ICC 14778 recorded significantly lowest 100 seed weight in gram. Similar findings were also obtained by Khourgami and Rafiee (2009) stated that the 100 seed weight in chickpea cultivars were significantly different. Hussain et al., (2015) also reported the similar results in chickpea under normal and rainfed condition.

\section{Yield (g)}

The effect of water stress on seed yield per plant (gm) in ten chickpea genotypes at harvest was demonstrated in table 3 and 4 Rabi season 2018-2019 the data showed that, with water stress, seed yield per plant significantly decreased in all ten genotypes. Under non-stress condition variety ICC 14778 $(25.36 \pm 0.15 \mathrm{gm})$ indicates maximum seed yield per plant closely followed by JG 11 $(21.36 \pm 0.25 \mathrm{gm})$, ICC $4958(20.48 \pm 0.14 \mathrm{gm})$ and Pusa-362 $(20.33 \pm 0.26 \mathrm{gm})$ whereas, the minimum seed yield per plant (gm) was recorded in ICC 867 (17.12 $\pm 0.27 \mathrm{gm})$. While under water stress condition ICC 14778 $(21.31 \pm 0.18 \mathrm{gm})$ produced maximum seed yield per plant closely followed by ICC 4958 (18.85 $\pm 0.03 \mathrm{gm}), \mathrm{JG} 11(17.92 \pm 0.38 \mathrm{gm})$ and Pusa-362 (17.49 \pm 0.25 gm) whereas, the minimum seed yield per plant (gm) was recorded in Digvijay (14.52 \pm 0.16 gm) (Figure 14).

The maximum reduction in Vishal (19.21\%) was reported and minimum reduction was reported in ICC 4958 (7.93\%) followed by ICC 867 (9.61\%) (Table 5). Therefore, the range from 17.12 to 25.36 and 14.52 to 21.31 under non-stress and stress condition, respectively.

In 2019-2020 under non-stress condition variety JG $11 \quad(24.3 \pm 0.08 \mathrm{gm})$ indicates maximum seed yield per plant closely followed by ICC 14778 (20.15 $\pm 0.39 \mathrm{gm})$, Pusa-362 [19.48 $\pm 0.11 \mathrm{gm}$ and Annigeri 1 $(19.46 \pm 0.27 \mathrm{gm})]$ whereas, the minimum seed yield per plant (gm) was recorded in Digvijay $(16.39 \pm 0.07 \mathrm{gm})$. While under water stress condition JG $11(19.49 \pm 0.11 \mathrm{gm})$ indicates maximum seed yield per plant closely followed by ICC 14778 (18.35 $\pm 0.18 \mathrm{gm})$, ICC 4958 (17.66 \pm 0.08 gm) and Pusa-362 (17.48 $\pm 1.68 \mathrm{gm})$ whereas, the minimum seed yield per plant (gm) was recorded in Digvijay 
$(13.16 \pm 0.07 \mathrm{gm})$ (Figure 15). The maximum reduction in Vishal $(20.46 \%)$ was reported and minimum reduction was reported in ICC $4958(8.10 \%)$ followed by ICC $867(8.60 \%)$ (Table 6). Therefore, the range from 17.12 to 25.36 and 14.52 to 21.31 under non-stress and stress condition, respectively.

The yield (g) per plant due to chickpea genotypes were statistically significant indicating considerable amount of genetic variation in yield potential. The highest yield per plant was recorded by genotypes ICC 14778 (25.36 gm) and JG 11 (24.3 gm) in 2018 and 2019, respectively. Results are conformity with the findings of Hussain et al., (2015) stated that the yield per plant $n$ chickpea cultivars were significantly different. Findings of similar genotypic variations were reported by Hegde et al., 2002 and Kumar et al., 2002. The results on yield plant $^{-1}$ indicate that the no-stress environment under normal irrigations gave highest yield plant $^{-\mathrm{I}}$.

In conclusion the yield data of tested genotypes showed a great variation in both non stress and stress conditions. In general, seed yield of genotypes under non irrigated condition was remarkably lower than irrigated condition. The seed yield per plant under non irrigated chickpea ranged from $13.74 \mathrm{~g}$ to $19.49 \mathrm{~g}$ and under irrigated condition 17.12 to $25.36 \mathrm{~g}$ per plant. The results of the present study indicated that ICC 4958 was determined as the best drought tolerant genotype followed by ICC 867 . In conclusion, genotypes or cultivars can be developed or selected on the basis of yield, early maturity under non stress conditions. Nevertheless, the genotypes identified in this study for traits related to higher productivity under non irrigated (drought stress) have important implications on accelerating the process of future breeding of adopted genotypes for drought prone area.

\section{Acknowledgement}

The authors want to express their sincere gratitude to the Indian Council of Agricultural Research (ICAR) for providing financial support through ICAR-SRF fellowship to corresponding author.

\section{References}

Ahmed, H., 2011. Evaluation of Chickpea (Cicer arietinum L.) Genotypes for Genetic Parameters and Drought Tolerance. Master of Science in Biology (Applied Genetics). Graduate Studies of Addis Ababa University.

Bidinger, F.R., V. Mahalaxmi, B.J. Talukdar, and G. Alagarswami. 1982. Improvement of drought resistance in pearl millet. pp. 45-49. In: Workshop on Principles and methods of crop improvement for drought resistance with emphasis on rice, 4-8 May 1981, International Rice Research Institute, Los Banos, Philippines.

Directorate of Economics and Statistics, DAC and FW 2019.

Evans, L.T. 1975. Crop Physiology, Cambridge university Press, London.

Ganjeali, A., H. Porsa, and A. Bagheri, 2011. Assessment of Iranian chickpea (Cicer arietinum L.) germplasm for drought tolerance. Agric. Water Manag. 98, 1477-1484.

Hegde, V.S., Yadav, S.S. and Kumar, J. 2002. Evaluation and selection of chickpea genotypes for adaptation to low input environments. Legume Res., 25 (1): 57-59

Hussain, N.M., Aslam, A. Ghaffar, M. Irshad and Naeem-ud-Din 2015. Chickpea genotypes evaluation for morpho-yield traits under water stress conditions. $J$. Anim. Plant Sci., 25: 206-211.

Karim, M. F., and Q. A. Fattah, 2006. Changes in yield of chickpea 
biocomponents of chickpea (Cicer arietinum L.) sprayed with potassium naphthenate and naphthalene acetic acid. Bangladesh J. Bot. 35, 39- 43.

Katerji, N., Wvan Hoorn, J., Hamdy, A., Mastrorilli, M., Oweis, T. and Malhotra, R.S. 2001. Response to soil salinity of two chickpea varieties differing in drought tolerance. Agricultural water management 50:8396.

Katiyar, R.P., Prasad, J., Singh, A.B. and Ram, K. 1977. Association analysis of grain yield and its components in segregating population of chickpea. Ind. J. Agric. Sci. 47 (1): 327-337.

Khourgami, A. and Rafiee, M. 2009. Drought stress, supplemental irrigation and plant densities in chickpea cultivars. African Crop Science Congress proceeds, 9: 141-143.

Kumar, J., and S. Abbo, 2001: Genetics of flowering time in chickpea and its bearing on productivity in semiarid environments. Adv. Agron. 72, 107138.

Kumar, S. 2016. Phasic Pre-treatment effect of $\mathrm{cd}, \mathrm{Zn}$ on activities of amaylase, protease and their uptake in seeds of Cicer arietinum CV C-235. Biotech today, 6(1): 18-20.

Kumar, S., Jeena, A.S. and Arora, P.P. 2002. Genetic variability and its importance for selection in chickpea. Agric. Sci. Digest, 22 (3): 215-216.

Mathur, R. and Mathur, M.L. 1996. Estimation of genetic parameter and inter-relationship of quantitative traits in chickpea. Madras Agricultural Journal, 83: 9-11.

Painawadee, M., S. Jogloy, T. Kesmala, C. Akkasaeng, and A. Patanothai. 2009. Heritability and correlation of drought resistance traits and agronomic traits in peanut (Arachis hypogaea L.). Asian J. Plant Science. 8:325-334.
Pandey, A. K., Sengar, R. S., Kumar, A., Chand, P., Yadav, R. and Vaishali 2018. Molecular Characterization of lentil (lens cultivars medikus) genotypes through SSR Markers. Biotech Today, 8:65-72

Panse, V.G. and Sukhatme, P.V. 1978. Statistical Methods for Agricultural Workers, Indian Council of Agricultural Research, New Delhi. $695 \mathrm{p}$

Passioura, J.B. 1996. Drought and drought tolerance. Plant Growth Regulation. 20: 79-83.

Pundir, R.P.S., Rao, N.K. and van den Maesen L.J.G. 1985 Distribution of qualitative traits in the world germplasm of chickpea (Cicer arietinum L.). Euphytica 34:697-703

Rahman, S.M.L. and Uddin, A.S.M.M. 2000. Ecological adaptation of chickpea (Cicer arietinum L.) to water stress. Grain yield, harvest index, flowering and maturity studies. Legume Research, 23: 1-8.

Rao, P P and Birthal, P S and Bhagavatula, S and Bantilan, M C S. 2010. Chickpea and Pigeonpea Economies in Asia: Facts, Trends and Outlook. Documentation. International Crops Research Institute for the Semi-Arid Tropics, Patancheru.

Rao, S.K. and Kumar, K.S. 2000. Analysis of yield factors in short duration chickpea (Cicer arietinum L.). Agriculture Science digest, 20: 65-67.

Reddy, N.R.N. and Ahlawat, I.P.S. 1998. Response of chickpea genotypes to irrigation and fertility under late sown conditions. Indian J. Agron., 43 (1): 95-101.

Saxena, N.P. and C. Johansen. 1990. Chickpea ideotypes for genetic enhancement of yield and yield stability in south Asia. pp.81-85. In: Chickpea in the Nineties: Proceedings 
of the Second International Workshop on Chickpea Improvement, 4-8 Dec 1989, ICRISAT Center, Patancheru, A.P, India.

Singh, K. B., R. S. Malhotra, M. H. Halila, E. J. Knights, and M. M. Verma, 1994. Current status and future strategy in breeding chickpea for resistance to biotic and abiotic stresses. In: F. J. Muehlbauer, and W. J. Kaiser (eds), Expending the Production and Use of Cool Season Food Legumes, 572591. Kluwer Academic Pub, Netherlands.

Subbarao, G.V., C. Johansen, A.E. Slinkard, R.C. N. Rao, N.P. Saxena, and Y. S. Chauhan. 1995. Strategies for improving drought resistance in grain legumes. Crit. Rev. Plant Sci. 14:469523.

Tahir, N. A.-R., and H. F. H. Karim, 2010. Impact of magnetic application on the parameters related to growth of chickpea (Cicer arietinum L.). Jordan J. Biol. Sci. 3, 175-184.

Tripathi, A.K., Pathak, M.M., Singh, K.P. and Singh, R.P. (1995). Path co-efficient analysis in chickpea. Ind. J. Pulses res, 8 (1): 71-72.

Varshney RK, Song C, Saxena RK, Azam S,
Yu S, Sharpe AG, Cannon S, Baek J, Rosen BD, Tar'an B, Millan T, Zhang $\mathrm{X}$, Ramsay LD, Iwata A, Wang Y, Nelson W, Farmer AD, Gaur PM, Soderlund C, Penmetsa RV, Xu C, Bharti AK, He W, Winter P, Zhao S, Hane JK, Carrasquilla-Garcia N, Condie JA, Upadhyaya HD, Luo MC, Thudi M, Gowda CL, Singh NP, Lichtenzveig J, Gali KK, Rubio J, Nadarajan N, Dolezel J, Bansal KC, Xu X, Edwards D, Zhang G, Kahl G, Gil J, Singh KB, Datta SK, Jackson SA, Wang J, Cook D.R. 2013. Draft genome sequence of chickpea (Cicer arietinum $\mathrm{L}$ ) provides a resource for trait improvement. Nat Biotechnol 31:240-246

Williams, P. C., and U. Singh, 1980. Nutritious quality and the evaluation of quality in breeding programmes. In: M. C. Saxena, and K. B. Singh, (eds), Chickpea, 329-356. CAB Int., UK.

Yadav, N.P., Sharma, C.M. and Heque, M.F. 2001. Correlation and regression study of seed yield and its components in chickpea. Journal of Research, Birsa Agricultural University, Ranchi, India, 13: $149-151$.

\section{How to cite this article:}

Shende, R. T., R. S. Sengar, Mukesh Kumar, Shalani Gupta, Gopal Singh and Singh, S. K. 2020. Evaluation of Yield and Yield Related Traits of Chickpea (Cicer arietinum L.) Genotypes under Water Stress Condition. Int.J.Curr.Microbiol.App.Sci. 9(12): 1960-1979. doi: https://doi.org/10.20546/ijcmas.2020.912.233 